A few words about the ratings are in order. Comparing this list with previously published lists it may be noted that the overall level is some 70 points lower than before. We recall that the Swedish rating list is a relative list, based on computer results against human beings in Swedish tournaments. However, in recent tournaments it has turned out that it is harder for computers to reach comparable results against human beings than some years ago. Closer investigation has led to the conclusion that we are faced with a "time-effect": chess-players are now much more used to play against computers than before. Therefore it is decided to calibrate the performances with games played during the two last years only.

We repeat the announcement by Mr. Göran Grottling of his up-to-date service, the Swedish Rating Service, offering Swedish Rating Lists ( 8 issues per year) for 120 SEK or alternate issues for 60 SEK. The equivalent in bank notes is also acceptable. If you go through Bank or Giro, please add 40 SEK to the amount stated, this being the equivalent of banking fees, so effectively rendering the payment net to the recipient. The lists will include all results and now cover 6 pages per issue, to be expanded soon. Dues are payable to SSDF, Swedish postal giro no 418772 - 0, accessible through any bank. The information is based on about 300 tournament games each month. All other information may be obtained by writing to Göran Grottling, Diabasvägen 3, S 43700 Lindome / Sweden.

\title{
THE CONFERENCE ADVANCES IN COMPUTER CHESS 6
}

\author{
London, UK \\ August 23-24, 1990
}

Call for Papers

The sixth conference in the Advances in Computer Chess series will be held in London on August 23-24, 1990. As with the previous conferences, held at three year intervals since 1975, there will be distinguished speakers from the field of computer chess, and the latest research results.

For the 1990 conference, it is hoped to arrange a human v. machine event involving the then-strongest chess computers against grandmasters, to take place alongside the conference. Also, David Levy's Second Computer Games Olympiad is scheduled to take place in London in the days prior to the chess conference (August 15-21, 1990).

Contributions are invited from authors who have new results or ongoing work to report on any aspect of computer chess or related theory.

All papers accepted for the conference will be published. Submissions should be sent to:

Don Beal

Department of Computer Science

Queen Mary \& Westfield College

Mile End Road

London E1 4NS

UK

to arrive not later than May 15, 1990. Papers should be in machine-readable form if at all possible MsWord is the preferred word processor. Authors will be notified of acceptance during July 1990. 